A. K. Dupree and A. O. Benz, Eds.

\title{
Circumstellar Disks around Young Solar-Type Stars
}

\author{
Inga Kamp \\ Leiden Observatory, PO Box 9513, 2300 RA Leiden, The Netherlands \\ Fatima Sammar \\ Leiden Observatory, PO Box 9513, 2300 RA Leiden, The Netherlands
}

\begin{abstract}
The chemistry of circumstellar disks around young (a few 10 Myr) solar-type stars is mainly driven by the strong UV radiation field of the central star. As a starting point for a detailed UV radiation field, the rocket and satellite observations of the solar chromosphere are used and scaled according to the time-dependent behaviour of stellar activity. The disk chemistry as well as dust and gas temperatures are then derived self-consistently from the model. The results of these calculations can be used for the identification of the most promising gas tracers as well as for the interpretation of present and future observations.
\end{abstract}

\section{Introduction}

The IRAS and ISO satellites revealed that a large fraction of nearby pre-main sequence solar-type stars is surrounded by cool dust. Near-infrared and submillimeter observations have shown that the dust is distributed in disk-like sometimes ring-like structures around the star (see Zuckerman 2001 for a recent review). However, in the literature the gas content of these protoplanetary disks is still under debate.

Current gas mass estimates neglect so far the possible existence of a chromosphere in solar-type stars. Ayres (1997) has shown that the ionizing ultraviolet flux from our Sun was much stronger in the past and evolved roughly as $t^{-1}$ with time. The aim of this work is to study the influence of a chromosphere on the disk chemistry and gas temperature in late phases of disk evolution, that is for tenuous disks with $L_{\mathrm{IR}} / L_{*}<0.1$.

\section{The model}

We use the stationary non-flaring disk models described by Kamp \& Bertoldi (2000) and Kamp \& van Zadelhoff (2001). These models solve for a prescribed density distribution the chemistry and the heating/cooling balance of the gas self-consistently. Recently, a few changes and improvements have been made to the Kamp \& van Zadelhoff (2001) models: (1) the inclusion of cosmic ray reactions, (2) a lower temperature of $20 \mathrm{~K}$ for freezing out of $\mathrm{CO}$ ice, (3) the inclusion of an escape probability formalism for the line photons (Tielens \& 

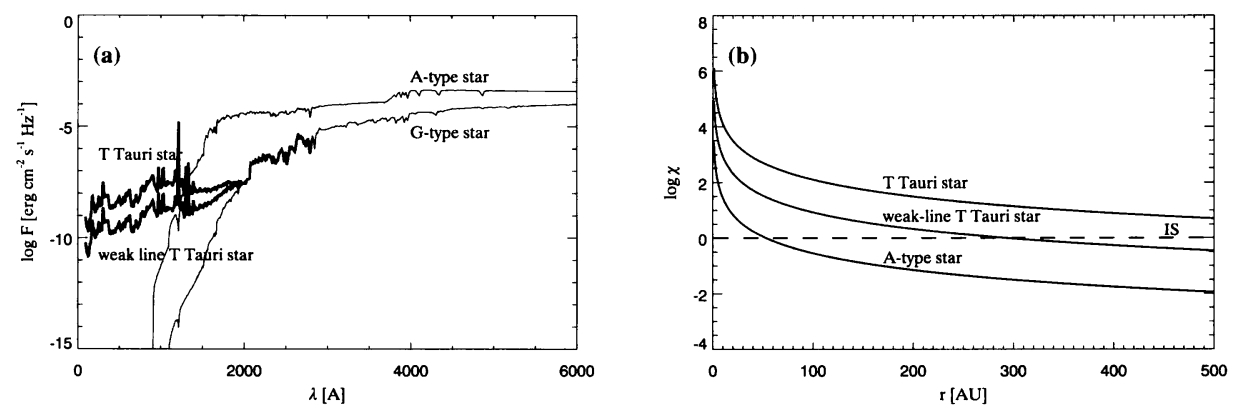

Figure 1. (a) Radiation fields of different stars: atmosphere of an A-type and G-type star and combined atmosphere and chromosphere of a weak-line $\mathrm{T}$ Tauri (70 Myr) and a $\mathrm{T}$ Tauri star (5 Myr). (b) Integrated UV flux normalized to the interstellar UV flux in the solar neighbourhood as a function of distance from the respective star.

Hollenbach 1985), and (4) statistical equilibrium for $\mathrm{C}$ II following the same approach as Kamp \& van Zadelhoff (2001) for O and CO. The first point is added for a more general use of the code. It does not affect the results described here, because the chemistry in our case is mostly driven by stellar photons. The escape probability formalism is necessary, because the main cooling lines, like the [O I] and CO lines, become optically thick. For a very conservative estimate of the escape probability, the line optical depth towards the star is used at each gridpoint to derive the escape probability of the line photon. Since the optical depth perpendicular to the disk is much lower than that, this is clearly a lower limit to the line cooling and hence an upper limit to the gas temperature.

\subsection{The chromospheric UV flux}

We follow the procedure outlined in Kamp \& Sammar (2003) and combine a stellar atmosphere model and current UV observations of our Sun to obtain the emergent spectrum of young-solar type stars of different age. The stellar model is an ATLAS9 atmosphere (Kurucz 1992) with an effective temperature of $5750 \mathrm{~K}$ and a $\log g$ of 4.5 . The solar UV observations are scaled according to the age of the respective star with a scaling factor $c=4900 /$ age(Myr) and a scaling law

$$
\log F_{\nu}(\text { star })=\log F_{\nu}(\text { sun })+\frac{\log c}{81.0}(2000-\lambda)
$$

where $F_{\nu}$ is given in erg $\mathrm{cm}^{-2} \mathrm{~s}^{-1} \mathrm{~Hz}^{-1}$ and $\lambda$ is in $\AA$. This relation holds for ages larger than $5 \mathrm{Myr}$. The resulting flux distribution for two different template stars is depicted in Fig. 1a. Figure 1b shows the strength of the UV radiation field (integral over the wavelength range 912 - $1110 \AA$ ) compared to the interstellar UV radiation field. It is evident that the chromospheric UV dominates the disk out to radii of at least $300 \mathrm{AU}$. 

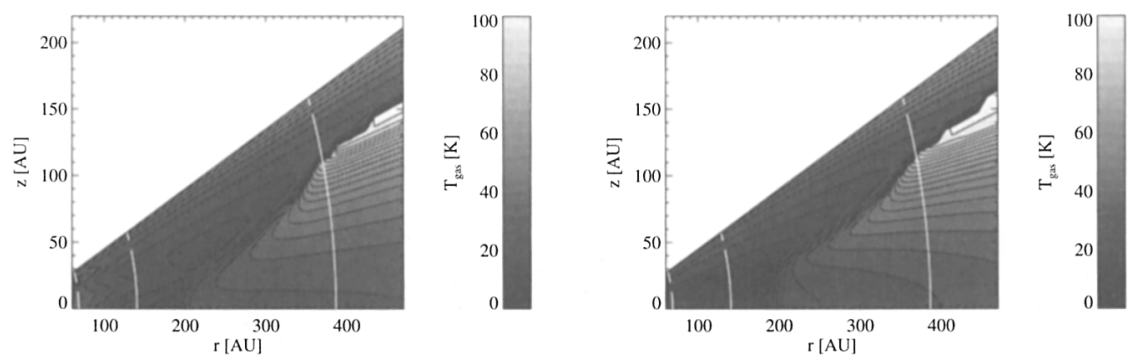

Figure 2. Gas temperatures in the weak-line T Tauri $\left(3.3 \mathrm{M}_{\oplus}\right)$ model with (a) and without (b) a chromosphere. The white contour lines indicate the dust temperature starting from $30 \mathrm{~K}$ at the outer edge and increasing inwards with a stepsize of $10 \mathrm{~K}$.

\section{The disk around a weak-line $\mathbf{T}$ Tauri stars}

Two model calculations are performed to illustrate the influence of the chromosphere on the gas temperature and chemistry in the disks around young solar-type stars. The disk model represents a weak-line $\mathrm{T}$ Tauri star with an age of $70 \mathrm{Myr}$ and a low disk mass of $3.3 \mathrm{M}_{\oplus}$, where the gas has already started to disperse, $\delta=M_{\text {dust }} / M_{\text {gas }}=0.07$. The disk extends from 60 to $470 \mathrm{AU}$ and we assume a mean dust grain size of $3 \mu \mathrm{m}$ and a corresponding UV absorption cross section of $5.3810^{-22} \mathrm{~cm}^{2}$ (H-atom) $)^{-1}$. The scale height $H_{0}=H / r$ is fixed to 0.15 and the radial power law exponent $\epsilon$ of the density distribution is 2.5 .

\subsection{The gas temperatures}

To illustrate the influence of a chromosphere, we model the gas temperature in this disk with and without a chromosphere. In the latter case, we take into account only the IS radiation field. As an approximation, we assume that this radiation penetrates the disk only from one side, namely from the inner radius.

Figure 2 illustrates that the core of the disk is slightly cooler $(\delta T \sim 20 \mathrm{~K})$ in the model without chromosphere. The reason for this is twofold: first, the photoelectric heating is smaller due to the lower UV flux; second, the chemistry in this model is different from the one with the chromosphere and hence $\mathrm{CO}$ and [C I] cooling are the dominant cooling processes. The chemistry in the disk model with chromosphere is subject to a higher UV flux. Therefore, The CO and $\mathrm{C}$ abundances are lower and [C II] cooling takes over in all except the innermost regions of the disk.

\subsection{The chemistry}

The chemistry in these tenuous disks is driven by the stellar UV photons. Before some details of the chemistry are discussed, we want to point out that often not even the interstellar UV radiation has been accounted for, if an upper limit to the gas mass is deduced from $\mathrm{CO}$ observations of these disks.

Figure 4 shows as an example the differences in $\mathrm{C}, \mathrm{C}$ II and $\mathrm{CO}$ abundances arising from the presence of a chromosphere around these stars. The higher UV flux, due to the stellar activity, enhances the photodissociation of CO and 

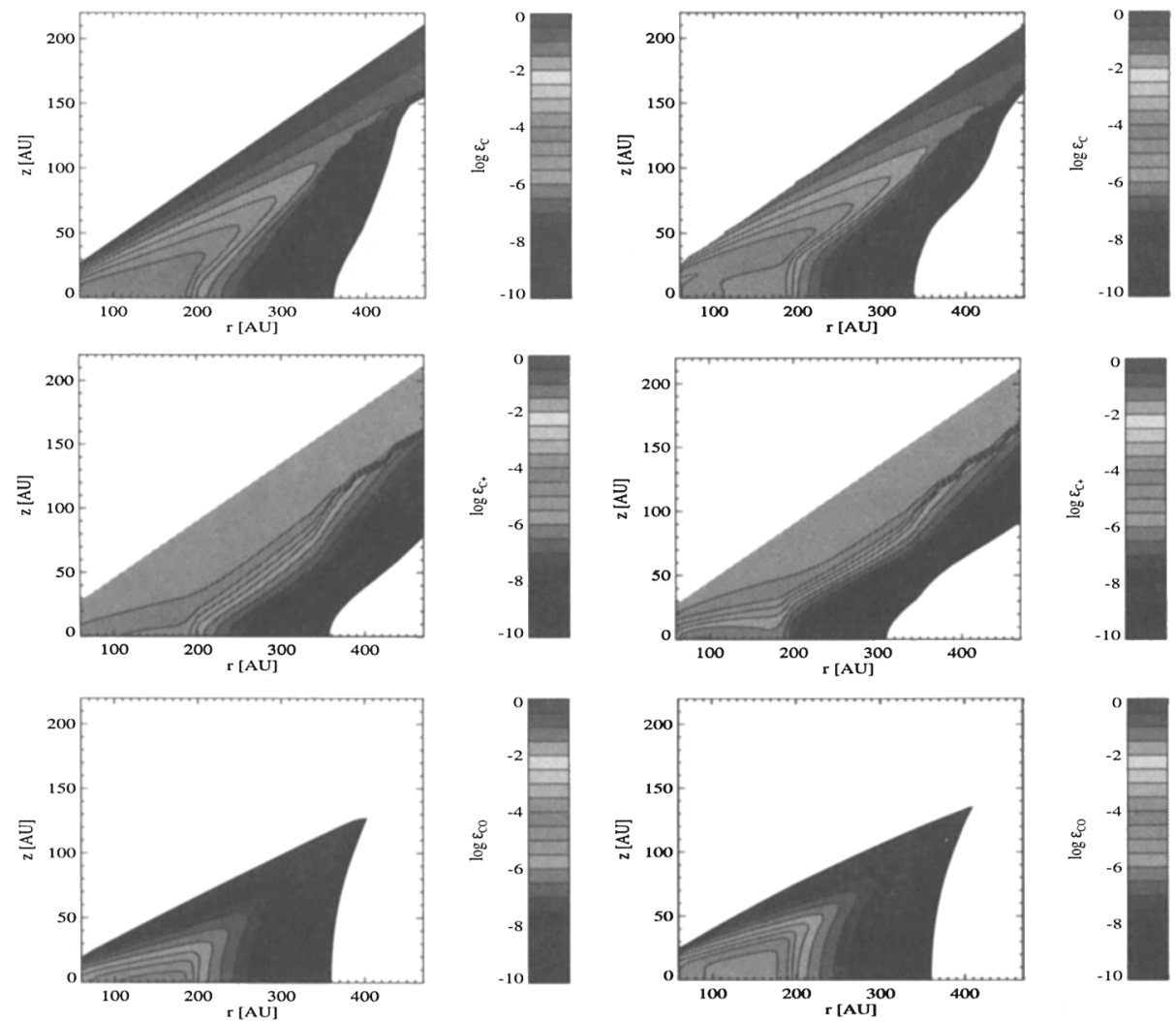

Figure 3. Chemistry in the weak-line T Tauri $\left(3.3 \mathrm{M}_{\oplus}\right)$ model with a chromosphere (left column) and without a chromosphere (right column). The contour plots show the $\mathrm{C}, \mathrm{C}$ II and $\mathrm{CO}$ abundances, $\log \epsilon_{\mathrm{el}}=n_{\mathrm{el}} / n_{\text {tot }}$.

ionizes carbon in the inner regions of the disk as well as above 1.5 scaleheights. At radii larger than $200 \mathrm{AU}, \mathrm{CO}$ freezes out onto the cold dust grains. Since the ice condensation depends on density, CO cannot freeze out in the upper layers of the disk and there $\mathrm{C}$ II remains the dominant carbon bearing species.

The influence of the chromospheric UV radiation can also be seen in Fig. 5, where the $\mathrm{H}$ and $\mathrm{H}_{2}$ abundances are displayed. The model with a chromosphere shows higher $\mathrm{H}$ abundances in the inner disk due to a shift of the $\mathrm{H} / \mathrm{H}_{2}$ transition.

\section{Conclusion}

These models show for the first time quantitatively the influence of a chromospheric radiation field on the disk around young solar-type stars and its implications for observations of the gas phase material.

The chromospheric UV photons lead to an increase in the photoelectric heating rate and hence to slightly warmer disks. The chemistry is mainly driven 

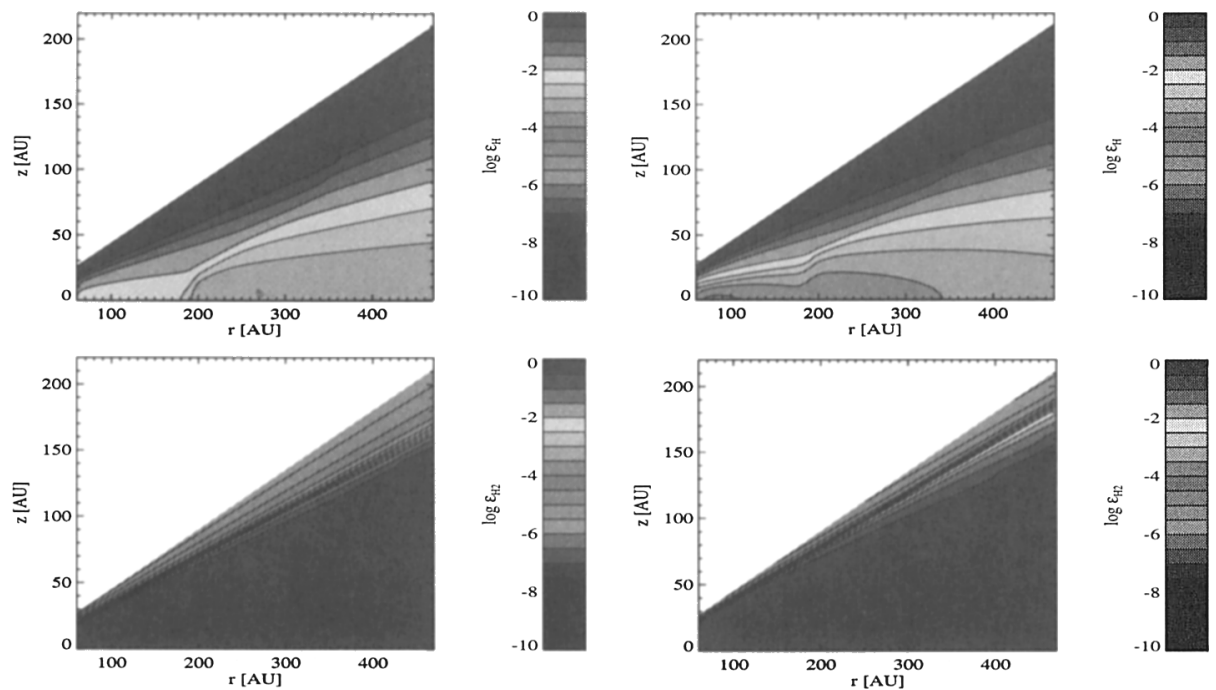

Figure 4. Chemistry in the weak-line T Tauri $\left(3.3 \mathrm{M}_{\oplus}\right)$ model with a chromosphere (left column) and without a chromosphere (right column). The contour plots show the $\mathrm{H}$ and $\mathrm{H}_{2}$ abundances, $\log \epsilon_{\mathrm{el}}=$ $n_{\mathrm{el}} / n_{\text {tot }}$.

by stellar UV photons and hence, the disk model without a chromosphere is more molecular than the one with a chromosphere.

The interstellar UV radiation field alone will already dissociate some of the CO in the inner disk. The outer disk parts are very cold and hence CO disappears from the gas phase by forming ice mantles on the dust grains. The non-detection of $\mathrm{CO}$ in tenuous disks around weak-line $\mathrm{T}$ Tauri stars does not necessarily imply a general gas deficit in these disks.

We suggest that $\mathrm{C}$ and $\mathrm{C}$ II are much better tracers for the gas content in the transition regime from protoplanetary disks to debris disks. The fine-structure lines of these species can be traced with current and future instrumentation like JCMT, APEX, SOFIA and Herschel (Kamp et al. 2003).

\section{References}

Ayres, T.R. 1997, JGR Planets 102, 1641

Kamp, I. \& Bertoldi, F. 2000, A\&A, 353, 276

Kamp, I. \& van Zadelhoff, G.-J. 2001, A\&A, 373, 641

Kamp, I., van Zadelhoff, G.-J., van Dishoeck, E.F., Stark, R. 2003, A\&A, 397, 1129

Kamp, I. \& Sammar, F. 2003, A\&A, submitted

Kurucz, R.L. 1992, Rev. Mex. Astron. Astrofis. 23, 181

Tielens, A.G.G.M., Hollenbach, D. 1985, ApJ, 291, 722

Zuckerman, B. 2001, ARA\&A, 39, 549 